\title{
UNIQUENESS AND QUASI-MEASURES ON THE GROUP OF INTEGERS OF A $p$-SERIES FIELD
}

\author{
WILLIAM R. WADE AND KAORU YONEDA
}

\begin{abstract}
Let $G$ be the group of integers of a $p$-series field and suppose that $S$ is a character series on $G$. If $N_{1}, N_{2}, \ldots$ is any sequence of integers and if $S_{p} N, \rightarrow 0$ a.e. on $G$, as $j \rightarrow \infty$, then $S$ will be the zero series provided $S$ never diverges unboundedly.
\end{abstract}

Let $G$ denote the group of integers of a $p$-series field, where $p$ is a prime $>2$. Thus, any element $\bar{x} \in G$ can be represented as a sequence $\left\{x_{i}\right\}_{i=0}^{\infty}$ with $0<x_{i}<p$ for each $i \geqslant 0$. Moreover, the dual group $\left\{\psi_{m}\right\}_{m=0}^{\infty}$ of $G$ can be described by the following process. If $m$ is a nonnegative integer with $m=\sum_{k=0}^{\infty} \alpha_{k} p^{k}, 0 \leqslant \alpha_{k}<p$ for each $k$, and if $\bar{x} \in G$ then

$$
\psi_{m}(\bar{x})=\prod_{k=0}^{\infty} \phi_{k}^{\alpha_{k}}(\bar{x}),
$$

where for each integer $k \geqslant 0$ and for each $\bar{x}=\left\{x_{i}\right\} \in G$, the function $\phi_{k}$ is defined by

$$
\phi_{k}(\bar{x})=\exp \left(2 \pi i x_{k} / p\right) .
$$

In the case that $p=2$, the group $G$ is the dyadic group introduced by Fine [2] and the functions $\left\{\psi_{m}\right\}_{m=0}^{\infty}$ are the Walsh-Paley functions. A detailed account of these groups and basic properties can be found in [5].

Denote the partial sums of a character series $S=\sum_{m=0}^{\infty} a_{m} \psi_{m}$ by

$$
S_{N}=\sum_{m=0}^{N-1} a_{m} \psi_{m}, \quad N=1,2, \ldots
$$

Vilenkin [6] has shown that if $S_{N} \rightarrow 0$ everywhere on $G$ as $N \rightarrow \infty$, then $S$ is the zero series, i.e., $a_{m}=0$ for $m=0,1, \ldots$ When $N$ is replaced by $p^{N}$ and convergence is relaxed on a countable subset of $G$, a growth condition is usually necessary to retain uniqueness. For example, in [8] we saw that if $S_{p^{N}}$ converges to an integrable $f$ on all but countably many points in $G$, and if $p^{-N} S_{p^{N}} \rightarrow 0$ everywhere on $G$ as $N \rightarrow \infty$, then $S$ is the $G$-Fourier series of $f$. The second hypothesis of this result cannot be relaxed at a single point $\bar{x}_{0} \in G$ where $\left|S_{p^{N}}\left(\bar{x}_{0}\right)\right| \rightarrow+\infty$ as

Received by the editors January 19, 1981.

1980 Mathematics Subject Classification. Primary 42C10, 42C25; Secondary 43A75, $12 B 99$.

Key words and phrases. Group of integers of a p-series field, uniqueness, Walsh functions, Egoroff's Theorem. 
$N \rightarrow \infty$. Indeed, if $D=\sum_{m=0}^{\infty} \psi_{m}$ represents the Dirichlet kernel on $G$, then since

$$
D_{p^{N}}(\bar{x})= \begin{cases}p^{N} & \text { if } \sum_{i=0}^{\infty} x_{i} p^{-i-1}<p^{-N}, \\ 0 & \text { otherwise, }\end{cases}
$$

it is clear that both $D_{p^{N}}(\bar{x})$ and $p^{-N} D_{p^{N}}(\bar{x})$ converge to zero for $\bar{x} \neq 0$ as $N \rightarrow \infty$ but $D$ is not the zero series. It is not yet known how far the first hypothesis can be relaxed (see [7]). One would expect that "convergence off a countable set" could be replaced with "convergence a.e." but even in the case $p=2$ this has not been done.

If one strengthens the second hypothesis to condition (4) below, then convergence a.e. can be used. Indeed, we shall prove the following result.

THEOREM. Suppose that $S=\sum_{m=0}^{\infty} a_{m} \psi_{m}$ and that $\left\{m_{j}\right\}_{j=1}^{\infty}$ is a subsequence of the natural numbers. If $S_{p^{m_{j}}} \rightarrow 0$ a.e. on $G$ as $j \rightarrow \infty$, and if

$$
\limsup _{j \rightarrow \infty}\left|S_{p^{m_{j}}}(\bar{x})\right|<\infty, \quad \bar{x} \in G,
$$

then $S$ is the zero series.

Techniques used to establish uniqueness for Walsh series fall into two categories: proof by a Haar series argument (e.g., [1]), and proof by differentiation (e.g., [2] and [4]). Neither of these techniques seem suited to prove the theorem above.

Our technique introduces a fresh viewpoint, and uses quasi-measures (defined below) as a crutch for carrying out the necessary calculations. Recall that the topology of $G$ has a base at 0 which consists of closed/open subgroups $G_{n}$ whose Haar measure $m\left(G_{n}\right)$ equals $p^{-n}, n \geqslant 0$. We shall denote $G_{0} \equiv G$ by $I(0,0)$, and for each integer $n>0$ we shall denote the cosets of $G_{n}$ by $I(k, n), 0<k<p^{n}$. The collection of sets $I(k, n), 0 \leqslant k<p^{n}, n=0,1, \ldots$, will be denoted by 9 . Observe once and for all that $I\left(k_{1}, n\right) \cap I\left(k_{2}, n\right)=\varnothing$ for $k_{1} \neq k_{2}$, that $m(I(k, n))=p^{-n}$, that (reordering if necessary)

$$
I(k, n)=\bigcup_{l=k p}^{k p+p-1} I(l, n+1)
$$

and that each $\psi_{l}$ is constant on each $I(k, n)$ when $l<p^{n}$. A set function $\mu$ defined on $\mathscr{G}$ is said to be a quasi-measure if

$$
\mu(I(k, n))=\sum_{l=k p}^{k p+p-1} \mu(I(l, n+1)) .
$$

Clearly, every Borel measure on $G$ is also a quasi-measure.

Fix integers $k$ and $n$ with $0 \leqslant k<p^{n}$. By an argument similar to that found in [3], one can show that if $\lambda$ is a Borel measure on $G$, and if $S$ is its Fourier-Stieltjes series, then

$$
\lambda(I(k, n))=\lim _{N \rightarrow \infty} \int_{I(k, n)} S_{N} d m .
$$

Since $\int_{I(k, n)} \psi_{l} d m=0$ for $l \geqslant p^{n}$ and since $S_{p^{n}}$ is constant on $I(k, n)$, it follows that $\lambda(I(k, n)) \equiv p^{-n} S_{p^{n}}(\bar{x})$ for any choice of $\bar{x} \in I(k, n)$. Thus we are led to associate 
with any character series $S$ the set function $\mu$ defined on 9 by

$$
\mu(I(k, n)) \equiv p^{-n} S_{p^{n}}(\bar{x}), \quad \bar{x} \in I(k, n) .
$$

A routine calculation (recall that the sum of $p$ th roots of unity is zero) establishes that this set function $\mu$ is a quasi-measure. Moreover, since the characters of $G$ are orthogonal it is clear that a necessary and sufficient condition for a character series $S$ to be zero is that its associated quasi-measure satisfies $\mu(I)=0$ for all $I \in \mathcal{G}$.

We are now prepared to prove the theorem. Indeed, if $S$ is the given series and if $\mu$ is its associated quasi-measure, we need only show that $\mu \equiv 0$. We shall actually show that $\mu(G)=0$. The same argument can be used to show that $\mu(I)=0$ for all $I \in \mathcal{G}$ and thus complete the proof.

We assume for simplicity that $m_{j}=j$. Fix $0<\varepsilon<1$. By Egoroff's Theorem we can choose a subset $E_{1}$ of $G$ such that $m\left(E_{1}\right)>1-\varepsilon$ and such that $S_{p^{N}}$ converges uniformly to zero on $E_{1}$, as $N \rightarrow \infty$. Thus, given $\varepsilon_{1}>0$ there exists an integer $N_{1}$ such that $\left|S_{p^{N_{1}}}(\bar{x})\right|<\varepsilon_{1}$ for $\bar{x} \in E_{1}$. But $S_{p^{N_{1}}}$ is actually constant on sets of the form $I\left(k, N_{1}\right)$ so there exists a subset $Z_{1}$ of the positive integers such that $k \in Z_{1}$ implies $\left|S_{p^{N_{1}}}(\bar{x})\right|<\varepsilon_{1}$ for $\bar{x} \in I\left(k, N_{1}\right)$.

Observe that if $\left|Z_{1}\right|$ represents the cardinality of $Z_{1}$ then

$$
\left|Z_{1}\right| \cdot p^{-N_{1}} \equiv \sum_{k \in Z_{1}} m\left(I\left(k, N_{1}\right)\right)>m\left(E_{1}\right)>1-\varepsilon .
$$

Thus $1-\left|Z_{1}\right| \cdot p^{-N_{1}}<\varepsilon$. Now, let $k_{1}$ be an integer which satisfies

$$
\left|\mu\left(I\left(k_{1}, N_{1}\right)\right)\right|=\max _{k \notin Z_{1}}\left|\mu\left(I\left(k, N_{1}\right)\right)\right|
$$

and observe that $S_{p^{N}} \rightarrow 0$ a.e. on $I\left(k_{1}, N_{1}\right)$ as $N \rightarrow \infty$. Thus, given $\varepsilon_{2}$ we can choose a subset $E_{2}$ of $I\left(k_{1}, N_{1}\right)$ such that $m\left(E_{2}\right)>(1-\varepsilon) p^{-N_{1}}$ and choose an integer $N_{2}$ such that $\left|S_{p^{N_{1}+N_{2}}}(\bar{x})\right|<\varepsilon_{2}$ for $\bar{x} \in E_{2}$.

Continuing in this manner, given any positive integer $j$ and $\varepsilon_{j}>0$ we can choose positive integers $N_{j}, k_{j}$, subsets $Z_{j}$ of natural numbers and $E_{j}$ of $G$ such that

$$
1-\left|Z_{j}\right| p^{-N_{j}}<\varepsilon
$$

$$
\text { if } k \in Z_{j} \text { then }\left|S_{p^{N_{1}}+\cdots+N_{j}}(\bar{x})\right|<\varepsilon_{j} \quad \text { for } \bar{x} \in I\left(k, N_{1}+\cdots+N_{j}\right)
$$

and

$$
\begin{aligned}
& \left|\mu\left(I\left(k_{j}, N_{1}+\cdots+N_{j}\right)\right)\right| \\
& =\max \left\{\left|\mu\left(I\left(k, N_{1}+\cdots+N_{j}\right)\right)\right|: k \notin Z_{j}\right. \\
& \left.\quad \text { and } I\left(k, N_{1}+\cdots+N_{j}\right) \subseteq I\left(k_{j-1}, N_{1}+\cdots+N_{j-1}\right)\right\} .
\end{aligned}
$$

To estimate $\mu(G)$, observe by (6) that $|\mu(G)|<T_{1}+\tilde{T}_{1}$ where

$$
T_{1}=\sum\left\{\left|\mu\left(I\left(k, N_{1}\right)\right)\right|: k \in Z_{1}\right\}
$$

and

$$
\tilde{T}_{1}=\sum\left\{\left|\mu\left(I\left(k, N_{1}\right)\right)\right|: 0<k<p^{N_{1}}, k \notin Z_{1}\right\} .
$$

To estimate $T_{1}$ we observe that $\left|Z_{1}\right| p^{-N_{1}}<1$ follows from the fact that there are at most $p^{N_{1}}$ sets of the form $I\left(k, N_{1}\right)$. Therefore, by (7) and (9) we have that $T_{1}<\varepsilon_{1}$. 
To estimate $\tilde{T}_{1}$ we apply (10) to conclude that

$$
\tilde{T}_{1} \leqslant\left(p^{N_{1}}-\left|Z_{1}\right|\right)\left|\mu\left(I\left(k_{1}, N_{1}\right)\right)\right| \text {. }
$$

Thus

$$
|\mu(G)| \leqslant \varepsilon_{1}+\left(p^{N_{1}}-\left|Z_{1}\right|\right)\left|\mu\left(I\left(k_{1}, N_{1}\right)\right)\right| .
$$

But (6), (7), (9), and (10) can be used to estimate $\left|\mu\left(I\left(k_{1}, N_{1}\right)\right)\right|$ by summing over those intervals $I\left(k, N_{1}+N_{2}\right)$ which are contained in $I\left(k_{1}, N_{1}\right)$ and separating the indices $k \in Z_{2}$ from $k \notin Z_{2}$. Again $\left|Z_{2}\right| p^{-N_{2}}<1$ follows from the fact that there are at most $p^{N_{2}}$ sets of the form $I\left(k, N_{1}+N_{2}\right)$ contained in $I\left(k_{1}, N_{1}\right)$.

If we continue breaking up $\left|\mu\left(I\left(k_{j}, N_{1}+\cdots+N_{j}\right)\right)\right|$ in this manner we arrive at the following inequality:

$$
\begin{aligned}
|\mu(G)| \leqslant & \varepsilon_{1}+\varepsilon_{2}\left(p^{N_{1}}-\left|Z_{1}\right|\right)+\cdots+\varepsilon_{j} \prod_{l=1}^{j-1}\left(p^{N_{l}}-\left|Z_{l}\right|\right) \\
& +\left|\mu\left(I\left(k_{j}, N_{1}+\cdots+N_{j}\right)\right)\right| \cdot \prod_{l=1}^{j}\left(p^{N_{l}}-\left|Z_{l}\right|\right),
\end{aligned}
$$

$j=1,2, \ldots$ Set $\varepsilon_{1}=\varepsilon / 2$ and for each integer $j>1$ set

$$
\varepsilon_{j}=2^{-j} \varepsilon\left(\prod_{l=1}^{j-1}\left(p^{N_{l}}-\left|Z_{l}\right|\right)\right)^{-1} .
$$

It follows from (8) and (11) that

$$
|\mu(G)| \leqslant \varepsilon\left(1-2^{-j}\right)+\varepsilon p^{N_{1}+\cdots+N_{j}}\left|\mu\left(I\left(k_{j}, N_{1}+\cdots+N_{j}\right)\right)\right| .
$$

Observe by construction that the collection of compact sets

$$
\left\{I\left(k_{j}, N_{1}+\cdots+N_{j}\right)\right\}_{j=1}^{\infty}
$$

is nested. Consesquently, we can choose a point $\bar{x}_{0}$ which belongs to $I\left(k_{j}, N_{1}+\cdots+N_{j}\right)$ for all integers $j \geqslant 1$. By (7), then, the estimate (12) becomes

$$
|\mu(G)| \leqslant \varepsilon+\varepsilon\left|S_{p^{N_{1}}+\cdots+N_{j}}\left(\bar{x}_{0}\right)\right| \text {. }
$$

Our simplifying assumption turns (4) into $\lim \sup _{N \rightarrow \infty} S_{p^{N}}\left(\bar{x}_{0}\right) \mid=A<\infty$. Thus

$$
|\mu(G)| \leqslant \varepsilon+A \varepsilon .
$$

In particular, if we let $\varepsilon \rightarrow 0$ we obtain $\mu(G)=0$, thus completing the proof of the theorem.

\section{BIBLIOGRAPHY}

1. F. G. Arutunjan and A. A. Talaljan, On uniqueness of Haar and Walsh series, Izv. Akad. Nauk SSSR Ser. Mat. 28 (1964), 1391-1408. (Russian)

2. N. J. Fine, On the Walsh functions, Trans. Amer. Math. Soc. 65 (1949), 372-414.

3. __ Fourier-Stieltjes series of Walsh functions, Trans. Amer. Math. Soc. 86 (1957), 246-255.

4. R. J. Lindahl, $A$ differentiation theorem for functions defined on the dyadic rationals, Proc. Amer. Math. Soc. 30 (1971), 349-352.

5. M. H. Taibleson, Fourier analysis on local fields, Math. Notes Series, Princeton Univ. Press, Princeton, N. J., 1975. 
6. N. Ya. Vilenkin, On a class of complete orthonormal systems, Izv. Akad. Nauk SSSR Ser. Mat. 11 (1947), 363-400. (Russian)

7. W. R. Wade, Growth conditions and uniqueness of Walsh series, Michigan Math. J. 24 (1976), $153-155$.

8. , Sets of uniqueness for the group of integers of a p-series field, Canad. J. Math. 31 (1979), $858-866$.

Department of Mathematics, University of Tennessee, Knoxville, Tennessee 37916

Department of Mathematics, University of Osaka Prefecture, Sakai, Osaka, Japan 